\title{
Short communication: Interrelationship between butyrate and glucose supply on butyrate and glucose oxidation by ruminal epithelial preparations
}

\author{
B. I. Wiese, ${ }^{*}$ P. Górka, ${ }^{*} \dagger$ T. Mutsvangwa, ${ }^{*}$ E. Okine, $\ddagger$ and G. B. Penner ${ }^{* 1}$ \\ *Department of Animal and Poultry Science, University of Saskatchewan, Saskatoon, SK, Canada, S7N 5A8 \\ †Department of Animal Nutrition and Feed Management, University of Agriculture in Krakow, al. Mickiewicza 24/28, 30-059 Krakow, Poland \\ ‡Department of Agricultural, Food and Nutritional Science, University of Alberta, AB, Canada, T6G 2P5
}

\begin{abstract}
The aim of this study was to determine whether dietary Na-butyrate supplementation affects butyrate and glucose oxidation by ruminal epithelial preparations and whether this effect can be acutely modulated by substrate (glucose and butyrate) supply. Eighteen Suffolk wether lambs (6 lambs/treatment) were blocked by body weight and, within block, randomly assigned to the control treatment $(\mathrm{CON})$ or to diets containing differing Na-butyrate inclusion rates (1.58 or $3.16 \%$ ) equating to 1.25 (B1.25), and 2.50\% (B2.50) butyrate on a dry matter basis, respectively. All lambs received their diet for a period of $14 \mathrm{~d}$. After dietary adaptation, lambs were killed and the ruminal epithelium was harvested from the ventral sac, minced finely, and used for in vitro incubations. Incubation medium contained either a constant concentration of glucose $(4 \mathrm{~m} M)$ with increasing butyrate concentrations $(0,5,15,25$, or 40 $\mathrm{m} M)$ or a constant butyrate concentration $(15 \mathrm{mM})$ with increasing glucose concentrations $(0,1,2,4$, or $8 \mathrm{mM}$ ) to allow for the evaluation of whether acute changes in the concentration of metabolic substrates affect the oxidation of glucose and butyrate. We observed no interactions between the in vivo and in vitro treatments. Increasing dietary butyrate supplementation linearly decreased glucose oxidation by ruminal epithelial preparations, but had no effect on butyrate oxidation. Increasing butyrate concentration in vitro decreased (cubic effect) glucose oxidation when butyrate concentration ranged between 5 and $15 \mathrm{mM}$; however, glucose oxidation was increased with a butyrate concentration of $40 \mathrm{~m} M$. Butyrate oxidation decreased (cubic effect) as glucose concentration increased from 1 to $4 \mathrm{mM}$; however, butyrate oxidation increased when glucose was included at $8 \mathrm{mM}$. The results of this study demonstrate that dietary butyrate supplementation can decrease glucose oxidation by the ruminal epithelium,
\end{abstract}

Received February 8, 2013.

Accepted May 21, 2013.

${ }^{1}$ Corresponding author: greg.penner@usask.ca but the relative supply of glucose and butyrate has a pronounced effect on substrate oxidation.

Key words: butyrate, glucose, ruminal epithelium, oxidation

\section{Short Communication}

The use of butyrate as a dietary supplement has received considerable interest in recent years (Guilloteau et al., 2009; Górka et al., 2011). Butyrate is considered to be the preferred energy substrate for ruminal epithelial cells. This is based on low portal recovery of butyrate compared with acetate and propionate when these short-chain fatty acids (SCFA) were infused into the washed reticulorumen (Kristensen et al., 2000a; Kristensen and Harmon, 2004a) and an inhibitory effect of butyrate on acetate and propionate activation and metabolism, at least in vitro (Ash and Baird, 1973; Harmon et al., 1991).

Intensive and preferential metabolism of butyrate over other SCFA by the ruminal epithelium has been reported in many in vivo and in vitro studies (Weigand et al., 1975; Kristensen et al., 2000a; Kristensen and Harmon, 2004a) and it is estimated that between 25 and $80 \%$ of the butyrate absorbed by the ruminal epithelium is metabolized to ketone bodies (Weigand et al., 1972; Kristensen et al., 2000b). However, ketogenesis does not contribute substantially to the cellular ATP supply (Kristensen and Harmon, 2004b). Extensive use of butyrate for ketogenesis and the low ATP yield arising from ketogenesis indicate that a substantial portion of the intracellular energy supply must be obtained from the oxidation of other substrates. Harmon (1986) showed that although butyrate decreases glucose uptake by rumen epithelium in vitro, glucose oxidation was not changed. Furthermore, Harmon (1986) and El-Kadi et al. (2009) observed an increase in glucose oxidation and lactate production from glucose by the ruminal epithelium when animals were fed high-concentrate diets. These data suggest that dietary fermentability and perhaps butyrate supply may alter butyrate and glucose oxidation by the ruminal epithelium.

This study aimed to determine whether dietary butyrate supplementation alters glucose and butyrate 
oxidation by the rumen epithelium and whether acute changes in substrate availability modulate substrate oxidation.

The study was conducted at the University of Saskatchewan and all experimental procedures involving animals were reviewed and approved by the University of Saskatchewan Animal Research Ethics Board (Protocol no. 20100021) before initiation of the study.

A detailed description of the study design has been published (Wilson et al., 2012). Briefly, 18 Suffolk wether lambs with an average initial BW of (mean \pm SD) $47.4 \pm 1.4 \mathrm{~kg}$ were provided $7 \mathrm{~d}$ to adapt to the housing $\left(2.25-\mathrm{m}^{2}\right.$ pens with rubber mats) and dietary conditions before starting the study. To ensure that DMI did not confound the results, lambs were stratified by BW and, within strata, assigned to 1 of 3 treatment diets with the amount of feed offered restricted to $3 \%$ of BW on a DM basis. The ingredient composition (\% of DM) of the diets consisted of barley silage (10), and a supplemental pellet (90) for the control treatment $(\mathbf{C O N})$. To provide dietary butyrate at 1.25 (B1.25) and $2.50 \%(\mathbf{B 2 . 5 0})$ on a DM basis, the supplement pellet was partially replaced (1:1 ratio) by Na-butyrate (Sigma Aldrich, St. Louis, MO) equating to dietary Nabutyrate inclusion rates of 1.58 and $3.16 \%$ (DM basis). The entire dose of the Na-butyrate was mixed with one-half of the silage allocation and offered at $0830 \mathrm{~h}$, with the remaining portion of the diet being offered at $1030 \mathrm{~h}$. This feeding strategy ensured that the whole dose of butyrate was consumed before feeding the remaining diet allocation. A similar feeding strategy was applied to CON lambs except that they only received an equal proportion of barley silage. All lambs received their treatments for $14 \mathrm{~d}$, which was deemed to be a sufficient amount of time, as marked changes in functional activity of the ruminal epithelium can be observed within $7 \mathrm{~d}$ (Etschmann et al., 2009).

Lambs were killed at $1000 \mathrm{~h}$, after receiving their initial feeding ( $0830 \mathrm{~h}$ feeding only), via captive bolt stunning and exsanguination. A $100-\mathrm{cm}^{2}$ piece of ruminal tissue was harvested from the ventral sac and the epithelium was gently separated from the underlying tissues. Epithelial sheets were washed and transported to the laboratory (for details, see Wilson et al., 2012). In the laboratory, the ruminal epithelium was blotted dry and minced finely. Approximately $250 \mathrm{mg}$ (exact weight recorded) of tissue was placed in $25-\mathrm{mL}$ Erlenmeyer flasks following the protocol described by Harmon et al. (1991) with minor modifications. To each flask, $2.75 \mathrm{~mL}$ of oxygenated buffer $(\mathrm{pH} 7.4$ and $39^{\circ} \mathrm{C}$; Table 1) containing either glucose $(4 \mathrm{mM})$ or butyrate $(15 \mathrm{mM})$ was added to determine glucose and butyrate oxidation, respectively. Furthermore, for the measurement of glucose oxidation, solutions contain- ing $0,55,165,275$, and $440 \mathrm{~m} M$ butyrate were prepared and spiked with $\mathrm{D}-\left[{ }^{14} \mathrm{C}(\mathrm{U})\right]$-glucose $(11.8 \mathrm{GBq} /$ mmol, PerkinElmer Inc., Woodbridge, ON, Canada). The respective radiolabeled stock solution $(252.4 \mu \mathrm{L})$ was then added to Erlenmeyer flasks (in triplicate) to achieve final butyrate concentrations of $0,5,15,25$, or $40 \mathrm{~m} M$, and $9 \mathrm{kBq}$ of ${ }^{14} \mathrm{C}$-glucose with a final glucose concentration of $4 \mathrm{~m} M$. For measurement of butyrate oxidation, solutions containing $0,11,22,44$, and $88 \mathrm{mM}$ glucose were prepared and spiked with $\left[1-{ }^{14} \mathrm{C}\right]$-butyrate (2.0 GBq/mmol, Moravek Biochemicals, Brea, CA). The radiolabeled stock solution $(252.4 \mu \mathrm{L})$ was then added to Erlenmeyer flasks (in triplicate) to achieve final glucose concentrations of $0,1,2,4$, or $8 \mathrm{~m} M$, and $9 \mathrm{kBq}$ of ${ }^{14} \mathrm{C}$-butyrate, achieving a final butyrate concentration of $15 \mathrm{mM}$ in all flasks. This design allowed us to evaluate whether increasing the concentration of glucose affects butyrate oxidation by ruminal epithelial preparations, and vice versa. The ranges of glucose and butyrate concentrations used were chosen to cover and extend beyond the ranges observed for plasma glucose and ruminal butyrate, respectively.

After radiolabeled stock solutions were added, the Erlenmeyer flasks were sealed with rubber stopper tops (model number 882310, Kimble Chase Life Science and Research Products LLC, Vineland, NJ) that were fitted with disposable center wells (part 882320, Kimble Chase Life Science and Research Products LLC). Flasks were placed in a shaking water bath (45 $\mathrm{rpm} / \mathrm{min}$ at $39^{\circ} \mathrm{C}$ ) and incubated for $1 \mathrm{~h}$, which was within the linear range of $\mathrm{CO}_{2}$ production measured in validation incubations before the start of the study and that reported by Harmon et al. (1991). In addition, triplicate flasks were prepared as blanks. Blanks were prepared in the same manner as all other flasks except they were not spiked with ${ }^{14} \mathrm{C}$-glucose or ${ }^{14} \mathrm{C}$-butyrate. Blanks were terminated at the start of the 1-h incubation period and were used to correct for background

Table 1. Chemical composition $(\mathrm{m} M)$ of experimental buffers ${ }^{1}$

\begin{tabular}{lcc}
\hline Chemical & $\begin{array}{c}\text { Glucose } \\
\text { oxidation }\end{array}$ & $\begin{array}{c}\text { Butyrate } \\
\text { oxidation }\end{array}$ \\
\hline $\mathrm{CaCl}_{2}$ & 2 & 2 \\
$\mathrm{MgCl}_{2}$ & 1 & 1 \\
$\mathrm{Na}_{2} \mathrm{HPO}_{4}$ & 2 & 2 \\
$\mathrm{NaH}_{2} \mathrm{PO}_{4}$ & 1 & 1 \\
$\mathrm{KCl}$ & 5 & 5 \\
$\mathrm{NaCl}$ & 30 & 30 \\
$\mathrm{Na}$-gluconate & 45 & 45 \\
$\mathrm{HEPES}$ & 15 & 15 \\
NaHCO & 25 & 25 \\
Glucose & 4 & \\
Na-butyrate & & 15
\end{tabular}

${ }^{1}$ Buffers contained penicillin G $(60 \mathrm{mg} / \mathrm{L})$, kanamycin sulfate $(100$ $\mathrm{mg} / \mathrm{L})$, and flurocytosine $(50 \mathrm{mg} / \mathrm{L})$. 
radioactivity measurements. Blanks including isotope but not including tissue were not used as the incubation medium contained antibiotics.

After the 1-h incubation period, all incubations were terminated by adding $1 \mathrm{~mL}$ of $12.1 \mathrm{~N} \mathrm{HCl}$, and flasks were placed on ice. Subsequently (within 5 min), 250 $\mu \mathrm{L}$ of Solvable solubilizer (Perkin Elmer, Waltham, MA) was injected into each center well and flasks were placed on ice for an additional $60 \mathrm{~min}$ to allow for the collection of ${ }^{14} \mathrm{CO}_{2}$. Each center well was then transferred into a 20-mL scintillation vial and $10 \mathrm{~mL}$ of scintillation cocktail (ReadySafe, Beckman Coulter Inc., Fullerton, CA) was added. Additionally, from each flask, $100 \mu \mathrm{L}$ of the incubation medium was placed in a $20-\mathrm{mL}$ scintillation vial and $10 \mathrm{~mL}$ of scintillation cocktail (Ready Safe Scintillation Cocktail, Beckman Coulter, Brea, CA) was added to determine activity of the medium using a liquid scintillation counter (model LS6500, Beckman Coulter). All rates of product formation were expressed per unit of tissue weight incubated in individual flasks.

Data were analyzed as a split-plot design accounting for the in vivo treatments (main plot; dietary Na-butyrate inclusion), in vitro treatments (subplot; glucose concentration or butyrate concentration), and the 2-way interaction. Block was originally included in the model, but initial analysis revealed no effects of block and thus it was removed from the model. Furthermore, we found no significant interactions between the in vivo (dietary butyrate supplementation) and in vitro (increasing concentration of butyrate or glucose in the medium) treatments on butyrate or glucose oxidation. Therefore, results for the dietary treatment and in vitro treatments are presented separately. Data sets for glucose and butyrate oxidation were analyzed independently. Polynomial contrasts were used to determine the effects of both the in vivo and in vitro treatment doses on butyrate and glucose oxidation. Differences were declared significant when $P<0.05$.

The effect of Na-butyrate supplementation on lamb BW and DMI, as well as rumen fermentation and plasma glucose and BHBA, are presented elsewhere (Wilson et al., 2012). Briefly, BW (mean \pm SEM; 47.4 $\mathrm{kg} \pm 1.41 ; P=0.92)$ and DMI during the final week of feeding $(1,447 \mathrm{~g} / \mathrm{d} \pm 44.3 ; P=0.64)$ were not different among treatments. Sodium-butyrate supplementation at $\mathrm{B} 2.50(52.4 \mathrm{~m} M)$ increased $(P=0.001)$ ruminal total SCFA concentration by $21 \mathrm{~m} M$ relative to CON $(31.3 \mathrm{mM})$, with B1.25 $(36.3 \mathrm{mM})$ being not different from CON. Additionally, the ruminal concentrations of butyric acid increased $(P<0.001)$ with Na-butyrate supplementation and were $5.6 \mathrm{~m} M$ for CON, $11.9 \mathrm{~m} M$ for B1.25, and $26.4 \mathrm{mM}$ for B2.50. Plasma glucose did not differ $(P=0.49)$ among treatments, with an average of $3.9 \mathrm{mM}$, but plasma BHBA increased $(P$ $<0.001$ ) with Na-butyrate supplementation from 10.9 $\mathrm{m} M$ for CON to 15.7 and $24.0 \mathrm{~m} M$ for B1.25 and B2.50, respectively.

Increasing dietary Na-butyrate supplementation linearly decreased glucose oxidation by ruminal epithelial preparations $(P=0.008$; Table 2$)$ but had no effect on butyrate oxidation $(P=0.49)$. The sparing effect of butyrate on glucose oxidation was further demonstrated as increasing the butyrate concentration in vitro resulted in a decrease (cubic effect, $P<0.001$; Figure 1 ) in glucose oxidation, but only when butyrate concentration ranged between 5 and $15 \mathrm{mM}$. Interestingly, glucose oxidation increased when the butyrate concentration increased from 0 to $5 \mathrm{mM}$ and was highest when butyrate concentration in the medium was $40 \mathrm{mM}$. Butyrate oxidation followed a similar response as glucose oxidation with a decrease in butyrate oxidation (cubic effect, $P=0.008$; Figure 2) as glucose concentration increased from 1 to $4 \mathrm{~m} M$; however, butyrate oxidation increased when glucose was included at $8 \mathrm{~m} M$, and butyrate oxidation increased when glucose concentration increased from 0 to $1 \mathrm{~m} M$ in the incubation medium.

Sodium-butyrate supplementation appears to be an effective strategy to stimulate intestinal and ruminal development in calves at weaning (Guilloteau et al., 2009; Górka et al., 2011), increase (quadratic) apparent totaltract digestibility of DM, OM, and NDF (Huhtanen et al., 1993), and reduce liver lipid content in dairy cattle during the postpartum phase of the transition period (DeFrain et al., 2006). Part of the response, at least for the stimulation of gastrointestinal development and reduction in liver lipid content, arising from butyrate supplementation can be explained by the preferential

Table 2. Effect of dietary butyrate supplementation on ${ }^{14} \mathrm{CO}_{2}$ production $[\mathrm{nmol} /(\mathrm{mg}$ of tissue $\times \mathrm{h})]$ from glucose and butyrate by ruminal epithelial preparations ${ }^{1}$

\begin{tabular}{|c|c|c|c|c|c|c|}
\hline \multirow[b]{2}{*}{${ }^{14} \mathrm{CO}_{2}$ production } & \multicolumn{3}{|c|}{ Dietary butyrate concentration } & \multirow[b]{2}{*}{ SEM } & \multicolumn{2}{|c|}{$P$-value } \\
\hline & $\mathrm{CON}$ & B1.25 & $\mathrm{B} 2.50$ & & Linear & Quadratic \\
\hline From glucose & 0.848 & 0.769 & 0.592 & 0.056 & 0.008 & 0.49 \\
\hline From butyrate & 0.855 & 1.713 & 0.721 & 0.488 & 0.85 & 0.15 \\
\hline
\end{tabular}

${ }^{1} \mathrm{CON}=$ control diet; B1.25 = dietary butyrate at $1.25 \%$ of DM; B2.50 = dietary butyrate at $2.50 \%$ of DM. 


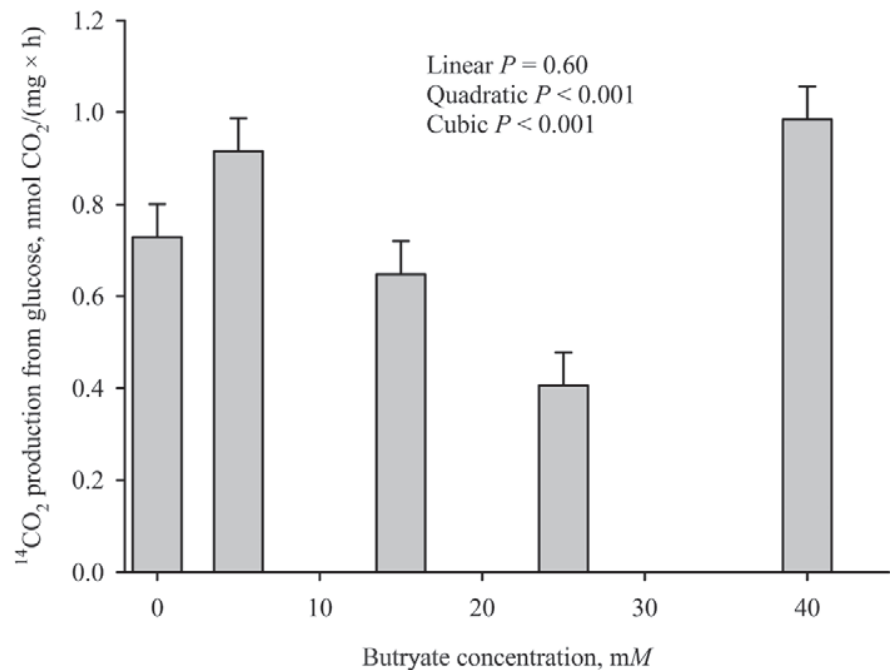

Figure 1. Effect of in vitro butyrate concentration on ${ }^{14} \mathrm{CO}_{2}$ production from glucose by ruminal epithelial preparations.

use of butyrate as an energy source by gastrointestinal tissues (Harmon et al., 1991; Baldwin and Jesse, 1992).

A major finding of the current study was that elevation of the ruminal butyrate concentration via dietary butyrate supplementation decreases glucose oxidation by ruminal epithelial preparations. In fact, glucose oxidation decreased by $30 \%$ when comparing $\mathrm{CON}$ with B2.50. Further supporting these results, we observed that glucose oxidation was also decreased when in vitro butyrate concentration was increased from 5 to 25 $\mathrm{m} M$, which reflects the physiological range of butyrate concentration in rumen fluid. It is unclear, however, why the highest concentrations of butyrate and glucose increased the oxidation of glucose and butyrate, respectively, but these findings may suggest that cell integrity was damaged, resulting in the leakage of cellular enzymes into the incubation medium. Moreover, it is important to note that the only oxidative substrates used in the incubation medium were glucose and butyrate. Thus, the low supply of anaplerotic substrates may have limited the citric acid cycle.

The sparing effect of SCFA on glucose oxidation by ruminal epithelial cells has been reported in many studies (Giesecke et al., 1979; Harmon et al., 1991) and butyrate reduced glucose oxidation by ruminal epithelial cells in newborn lambs (Baldwin and Jesse, 1992). This study, to our knowledge, is the first showing that dietary butyrate supplementation may be an effective strategy to decrease glucose utilization by the reticulo-ruminal epithelium. However, using tissue from the same lambs, Wilson et al. (2012) reported lower rates of butyrate flux across the isolated ruminal epithelia with dietary butyrate supplementation, suggesting that butyrate supply may also be affected. Considering the finding

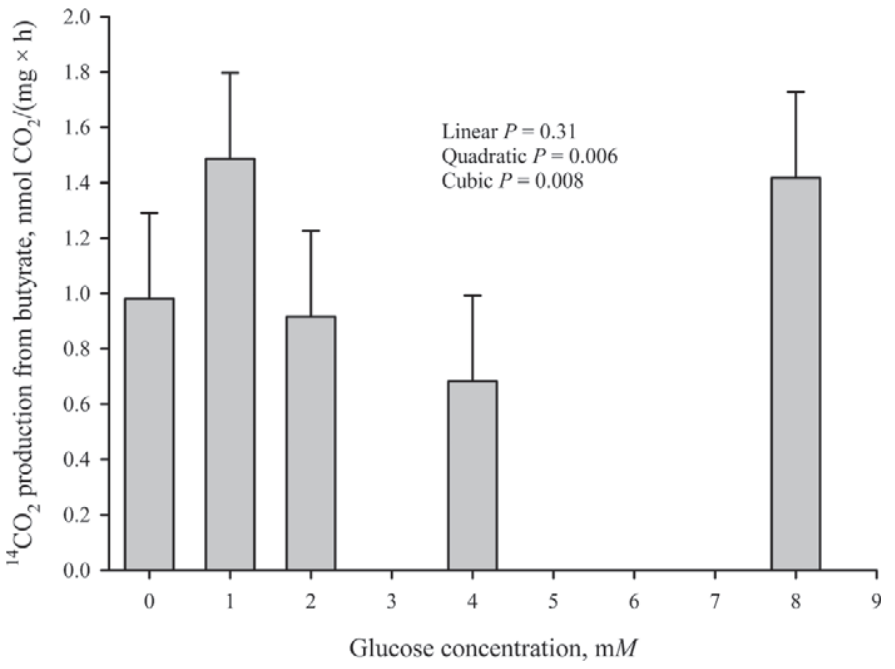

Figure 2. Effect of in vitro glucose concentration on ${ }^{14} \mathrm{CO}_{2}$ production from butyrate by ruminal epithelial preparations.

of lower rates of absorption, and high serum BHBA by Wilson et al. (2012), and that butyrate oxidation was not affected by dietary butyrate supplementation in the present study, it appears that butyrate supplementation does not affect butyrate oxidation but helps to reduce glucose oxidation by the ruminal epithelium. Future research is needed to evaluate whether butyrate supplementation affects glucose uptake by the portaldrained viscera.

Catabolic pathways for butyrate include ketogenesis and oxidative metabolism via $\beta$-oxidation and the citric acid cycle. However, ketogenesis only equates to a small ATP yield (0 to 3 ATP equivalents/mole of butyrate) depending on the relative proportion of acetoacetate and BHBA produced (Kristensen and Harmon, 2004b). Glucose modulates butyrate catabolism by diverting butyrate from oxidative pathways toward ketogenesis (Giesecke et al., 1979; Beck et al., 1984). However, this shift in metabolism also implies that the relative contribution of butyrate toward meeting the cellular energy supply decreases when glucose and glucogenic substrates are present. Indeed, the results of the current study support that increasing the glucose supply decreases butyrate oxidation by ruminal epithelial preparations.

Metabolic flexibility of ruminal epithelium has been demonstrated (Baldwin and Jesse, 1992; El-Kadi et al., 2009) and supports the results of the current study, as acute changes in glucose and butyrate availability greatly affected the oxidation of butyrate and glucose, respectively. An acute ability of the ruminal epithelium to adapt and utilize available substrates may be especially important following feeding. For example, Sehested et al. (1997) reported that butyrate as well 
as $\mathrm{Na}^{+}$and $\mathrm{Cl}^{-}$transport across rumen epithelium was increased only when the feeding strategy greatly increased SCFA concentration in the rumen. On the other hand, whereas the glucose concentrations used in this study aimed to represent the physiological range for serosal glucose concentration, the potential effect of ruminal glucose on the ruminal epithelial energy supply and function cannot be excluded (Aschenbach et al., 2000, 2011; Penner et al., 2011). Although ruminal glucose concentration is generally low $(<0.7$ $\mathrm{m} M)$, high-grain feeding may result in ruminal glucose concentrations up to $12 \mathrm{~m} M$ (Owens et al., 1998), and even moderate grain feeding ( $45 \%$ barley grain on DM basis) can yield values as high as $2 \mathrm{~m} M$ (Ametaj et al., 2010). It is clear that the ruminal epithelium has the capability to extract glucose from both arterial and luminal sources and that acute increases in the apical absorptive capability have been shown (Aschenbach et al., 2000). Thus, conditions where ruminal glucose concentration is increased may consequently decrease butyrate oxidation.

Altogether, the results of this study demonstrated that in vivo butyrate supplementation and increases in the concentration of butyrate in vitro decrease glucose oxidation by ruminal epithelial preparations. However, increasing the supply of glucose decreased butyrate oxidation, confirming the metabolic flexibility of the ruminal epithelium.

\section{ACKNOWLEDGMENTS}

Funding for the project was provided by the Presidents NSERC Fund at the University of Saskatchewan. Stipend support for B. I. Wiese was provided from the NSERC-USRA program. The authors thank D. J. Wilson (University of Saskatchewan, Saskatoon, SK, Canada) for help with animal care and sample collection.

\section{REFERENCES}

Ametaj, B. N., Q. Zebeli, F. Saleem, N. Psychogios, M. J. Lewis, S. M. Dunn, J. G. Xia, and D. S. Wishart. 2010. Metabolomics reveals unhealthy alterations in rumen metabolism with increased proportion of cereal grain in the diet of dairy cows. Metabolomics 6:583-594.

Aschenbach, J. R., G. B. Penner, F. Stumpff, and G. Gäbel. 2011. Ruminant Nutrition Symposium: Role of fermentation acid absorption in the regulation of ruminal pH. J. Anim. Sci. 89:1092-1107.

Aschenbach, J. R., H. Wehining, M. Kurze, E. Schaberg, H. Nieper, G. Bruckhardt, and G. Gäbel. 2000. Functional and molecular biological evidence of SGLT-1 in the ruminal epithelium of sheep. Am. J. Physiol. Gastrointest. Liver Physiol. 279:G20-G27.

Ash, R., and G. D. Baird. 1973. Activation of volatile fatty acids in bovine liver and rumen epithelium. Biochem. J. 136:311-319.

Baldwin, R. L. VI, and B. W. Jesse. 1992. Developmental changes in glucose and butyrate metabolism by isolated sheep ruminal cells. J. Nutr. 122:1149-1153.
Beck, U., B. Emmanuel, and D. Giesecke. 1984. The ketogenic effect of glucose in rumen epithelium of ovine (Ovis aries) and bovine (Bos taurus) origin. Comp. Biochem. Physiol. B 77:517-521.

DeFrain, J. M., A. R. Hippen, K. F. Kalscheur, and D. J. Schingoethe 2006. Feeding lactose to increase ruminal butyrate and the metabolic status of transition dairy cows. J. Dairy Sci. 89:267-276.

El-Kadi, S. W., R. L. Baldwin VI, K. R. McLeod, N. E. Sunny, and B. J. Bequette. 2009. Glutamate is the major anaplerotic substrate in the tricarboxylic acid cycle of isolated rumen epithelial and duodenal mucosal cells from beef cattle. J. Nutr. 139:869-875.

Etschmann, B., A. Suplie, and H. Martens. 2009. Change of ruminal sodium transport in sheep during dietary adaptation. Arch. Anim. Nutr. 63:26-38.

Giesecke, D., U. Beck, S. Wiesmayr, and M. Stangassinger. 1979. Effect of rumen epithelial development on metabolic activities and ketogenesis by the tissue in vitro. Comp. Biochem. Physiol. B 62:459-463.

Górka, P. Z. M. Kowalski, P. Pietrzak, A. Kotunia, W. Jagusiak, J. J. Holst, R. Guilloteau, and R. Zabielski. 2011. Effect of method of delivery of sodium butyrate on rumen development in newborn calves. J. Dairy Sci. 94:5578-5588.

Guilloteau, P., R. Zabielski, J. C. David, J. W. Blum, J. A. Morisset, M. Biernat, J. Woliński, D. Laubitz, and Y. Hamon. 2009. Sodiumbutyrate as a growth promoter in milk replacer formula for young calves. J. Dairy Sci. 92:1038-1049.

Harmon, D. L. 1986. Influence of dietary energy intake and substrate addition on the in vitro metabolism of glucose and glutamine in rumen epithelial tissue. Comp. Biochem. Physiol. B 85:643-647.

Harmon, D. L., K. L. Gross, R. C. Krehbiel, K. K. Kreikemeier, M. L. Bauer, and R. A. Britton. 1991. Influence of dietary forage and energy intake on metabolism and acyl-CoA synthetase activity in bovine ruminal epithelial tissue. J. Anim. Sci. 69:4117-4127.

Huhtanen, P., H. Miettinen, and M. Ylinen. 1993. Effect of increasing ruminal butyrate on milk yield and blood constituents in dairy cows fed a grass silage based diet. J. Dairy Sci. 76:1114-1124.

Kristensen, N. B., and D. L. Harmon. 2004a. Effect of increasing ruminal butyrate absorption on splanchnic metabolism of volatile fatty acids absorbed from the washed reticulorumen of steers. J. Anim. Sci. 82:3549-3559.

Kristensen, N. B., and D. L. Harmon. 2004b. Splanchnic metabolism of volatile fatty acids absorbed from the washed reticulorumen of steers. J. Anim. Sci. 82:2033-2042.

Kristensen, N. B., S. G. Pierzynowski, and A. Danfaer. 2000a. Net portal appearance of volatile fatty acids in sheep intraruminally infused with mixtures of acetate, propionate, isobutyrate, butyrate, and valerate. J. Anim. Sci. 78:1372-1379.

Kristensen, N. B., S. G. Pierzynowski, and A. Danfaer. 2000b. Portaldrained visceral metabolism of 3-hydroxybutyrate in sheep. J. Anim. Sci. 78:2223-2228.

Owens, F. N., D. S. Secrist, J. W. Hill, and D. R. Gill. 1998. Acidosis in cattle: A review. J. Anim. Sci. 76:275-286.

Penner, G. B., M. A. Steele, J. R. Aschenbach, and B. W. McBride 2011. Ruminant Nutrition Symposium: Molecular adaptation of ruminal epithelia to highly fermentable diets. J. Anim. Sci. 89:1108-1119

Sehested, J., A. Basse, J. B. Andersen, L. Diernaes, P. D. Moller, E. Skadhauge, and O. Aaes. 1997. Feed-induced changes in transport across the rumen epithelium. Comp. Biochem. Physiol. A Physiol. 118:385-386.

Weigand, E., J. W. Young, and A. D. McGilliard. 1972. Extent of butyrate metabolism by bovine ruminoreticulorumen epithelium and relationship to absorption rate. J. Dairy Sci. 55:589-597.

Weigand, E., J. W. Young, and A. D. McGilliard. 1975. Volatile fatty acid metabolism by rumen mucosa from cattle fed hay or grain. J. Dairy Sci. 58:1294-1300.

Wilson, D. J., T. Mutsvangwa, and G. B. Penner. 2012. Supplemental butyrate does not enhance the absorptive or barrier functions of the isolated ovine ruminal epithelia. J. Anim. Sci. 90:3153-3161. 